\title{
Validation of the Distance Visibility Algorithm (DiVA) and the Impact of the Mesoscale Approximation to Mine Warfare Applications
}

\author{
Alan Weidemann \\ Ocean Optics Section Code 7333, Naval Research Laboratory \\ Stennis Space Center, MS 39529-5004 \\ phone: (218) 688-6232, fax: (228) 688-4149, e-mail: alanw@nrlssc.navy.mil \\ Vladimir Haltrin \\ Ocean Optics Section Code 7333, Naval Research Laboratory \\ Stennis Space Center, MS 39529-5004 \\ phone: (218) 688-4528, fax: (228) 688-4149, e-mail: Haltrin@nrlssc.navy.mil \\ Walton McBride \\ Planning Systems Incorporated \\ Stennis Space Center, MS 39529-5004 \\ phone: (218) 689-8458, fax: (218) 689-XXXX, e-mail: wmcbride@psistennis.com \\ Award Number: N0001402WX21200 \\ http://www7333.nrlssc.navy.mil
}

\section{LONG-TERM GOALS}

The Navy has a long-term need for diver and camera visibility predictions in order to improve mine and expeditionary warfare applications. The goal of this research is to evaluate existing and emerging diver/camera visibility models prior to implementation into fleet operations. This research includes the recommendation of instrumentation and models that can be incorporated into Navy survey and battle groups for prediction and on-scene determination of diver/camera visibility operations.

\section{OBJECTIVES}

The Distance Visibility Algorithm (DiVA) is a model proposed by HOBILabs Inc., to predict diver and camera visibility for mesoscale targets. Originally the diver/camera visibility was to be dependent on measurements made with only one instrument (a-ßeta; HOBILabs Inc.,) and a lighting function. The model now is more complex with beam attenuation, $c$, required as well as target properties and the a$\beta$ eta measurement. The objectives of this research is to evaluate the mesoscale size constraint of DiVA, to determine the model's assumptions, check for internal consistencies, and then compare DiVA to more classical formulations of visibility such as contrast transmittance theory (CTT). This research also evaluates potential improvements that can be made using a complex model of diver visibility over the classical CTT approaches.

\section{APPROACH}

The evaluation of visibility models require an in-depth analysis of the model theory, a definition of the parameters used, and validation of the predicted visibility using in-situ measurements. The theoretical approach was to compare the Modulation Transfer Function formulation of contrast used by DiVA 


\section{Report Documentation Page}

Form Approved

OMB No. 0704-0188

Public reporting burden for the collection of information is estimated to average 1 hour per response, including the time for reviewing instructions, searching existing data sources, gathering and maintaining the data needed, and completing and reviewing the collection of information. Send comments regarding this burden estimate or any other aspect of this collection of information,

including suggestions for reducing this burden, to Washington Headquarters Services, Directorate for Information Operations and Reports, 1215 Jefferson Davis Highway, Suite 1204, Arlington

VA 22202-4302. Respondents should be aware that notwithstanding any other provision of law, no person shall be subject to a penalty for failing to comply with a collection of information if it

does not display a currently valid OMB control number.

\begin{tabular}{|c|c|}
\hline $\begin{array}{l}\text { 1. REPORT DATE } \\
\mathbf{3 0} \text { SEP } \mathbf{2 0 0 2}\end{array}$ & $\begin{array}{l}\text { 3. DATES COVERED } \\
\mathbf{0 0 - 0 0 - 2 0 0 2} \text { to 00-00-2002 }\end{array}$ \\
\hline \multirow{3}{*}{$\begin{array}{l}\text { Validation of the Distance Visibility Algorithm (DiVA) and the Impact of } \\
\text { the Mesoscale Approximation to Mine Warfare Applications }\end{array}$} & 5a. CONTRACT NUMBER \\
\hline & 5b. GRANT NUMBER \\
\hline & 5c. PROGRAM ELEMENT NUMBER \\
\hline \multirow[t]{3}{*}{ 6. AUTHOR(S) } & 5d. PROJECT NUMBER \\
\hline & 5e. TASK NUMBER \\
\hline & 5f. WORK UNIT NUMBER \\
\hline $\begin{array}{l}\text { 7. PERFORMING ORGANIZATION NAME(S) AND ADDRESS(ES) } \\
\text { Ocean Optics Section Code 7333, Naval Research Laboratory,,Stennis } \\
\text { Space Center,,MS, } 39529\end{array}$ & $\begin{array}{l}\text { 8. PERFORMING ORGANIZATION } \\
\text { REPORT NUMBER }\end{array}$ \\
\hline \multirow[t]{2}{*}{ 9. SPONSORING/MONITORING AGENCY NAME(S) AND ADDRESS(ES) } & 10. SPONSOR/MONITOR'S ACRONYM(S) \\
\hline & $\begin{array}{l}\text { 11. SPONSOR/MONITOR'S REPORT } \\
\text { NUMBER(S) }\end{array}$ \\
\hline
\end{tabular}

12. DISTRIBUTION/AVAILABILITY STATEMENT

Approved for public release; distribution unlimited

13. SUPPLEMENTARY NOTES

14. ABSTRACT

The Navy has a long-term need for diver and camera visibility predictions in order to improve mine and expeditionary warfare applications. The goal of this research is to evaluate existing and emerging diver/camera visibility models prior to implementation into fleet operations. This research includes the recommendation of instrumentation and models that can be incorporated into Navy survey and battle groups for prediction and on-scene determination of diver/camera visibility operations.

15. SUBJECT TERMS

16. SECURITY CLASSIFICATION OF:

a. REPORT

unclassified b. ABSTRACT unclassified c. THIS PAGE unclassified
17. LIMITATION OF ABSTRACT

Same as Report (SAR)
18. NUMBER OF PAGES

19a. NAME OF RESPONSIBLE PERSON

7 
with the contrast transmittance theory (CTT) used by previous investigators ( Duntley, 1963). The theoretical analysis between DiVA and CTT includes: 1) comparison of CTT and DiVA contrast definitions and their relationship, 2) comparison of CTT and DiVA maximum horizontal visibility equations, 3 ) investigation of the meaning of $K_{L}$, the radiance attenuation parameter used by DiVA, and 4 ) the sensitivity of the parameters required in DiVA. The DiVA model uses an analytical expression for the light field which, in theory, should improve visibility estimates, but the extent of this improvement relative to the uncertainties in target size, range, and reflectivity requires evaluation. The issue is what additional information, if any, is provided by the DiVA model and how one could use it.

Field data was collected to evaluate the "mesoscale" condition for visibility models and included a clear and turbid water environment. For DiVA evaluation, measurements of the parameters needed were made with the a- $\beta$ eta and c- $\beta$ eta instruments. To compare with other models and to check for instrumentation capabilities, the beam transmission, absorption, and backscattering measurements were also made with other commercially available instruments. Visibility measurements were made with Navy divers and with digital imagery taken of black, as well as, black and white targets. The digital imagery and the measured optical properties were used in a Graphical User Interface specifically designed to compare CTT and DiVA results and to evaluate $K_{L}$ versus the attenuation coefficient measured by the a- $\beta$ eta $\left(K_{a \cdot e t a}\right)$.

\section{WORK COMPLETED}

A theoretical comparison between CTT and DiVA formulations for maximum diver visibility is near completion. This work includes a comparison of the definitions used between DiVA and the CTT, and a comparison of $K_{L}$ used by DiVA, and $c$. These analyses were required to determine whether the MTF formulation, at a mesoscale level, significantly improves visibility estimates. The utility of having the radiance distribution provided by DiVA was also evaluated.

In-situ data was collected and analyzed from two exercises; a clear water condition off of Pensacola, FL, and turbid water condition off of Gulfport, MS. Visibility measurements of black, as well as, black and white targets (plate and spheres) submerged at depth were made by Navy Reserve divers (from the Gauging Littoral Optics Program, CNMOC and ONR). These targets were selected to reproduce the methodology used by HOBILabs Inc., in DiVA development. The in-situ data also included digital imagery of targets at increasing distances so that the radiance of the target relative to the background could be determined. The oceanographic measurements include a- $\beta$ eta, c- $\beta$ eta, ac-9 (Wetlabs Inc.,), CTD, sky and sea-surface conditions, and Secchi disc range.

\section{RESULTS}

Comparison of CTT and DiVA contrast definitions and their relationship: Although defined differently, there is a straightforward relationship between both contrast definitions.

$$
C_{D i V A}(0)=\frac{L_{T O}-L_{B O}}{L_{T O}+L_{B O}} \quad \text { and } \quad C_{C T T}(0)=\frac{L_{T O}-L_{B O}}{L_{B O}}
$$

where $\mathrm{C}_{D i V A}(0)$ and $\mathrm{C}_{C T T}(0)$ are the inherent contrasts, and $\mathrm{L}_{T O}$, and and $\mathrm{L}_{B O}$ are the inherent radiances of the target and the background respectively. Equating the numerators of both contrast definitions:

$$
\left(L_{T O}+L_{B O}\right) C_{D i V A}(0)=C_{C T T}(0) L_{B O}
$$

it can be shown that 


$$
C_{C T T}(0)=\frac{2 C_{D i V A}(0)}{1-C_{D i V A}(0)} \quad \text { and } \quad C_{D i V A}(0)=\frac{C_{C T T}(0)}{2+C_{C T T}(0)} .
$$

The above relationships for target contrast are also valid for the contrast threshold (the threshold beyond which one can no longer discern an object against its background; $\mathrm{C}_{m C T T}$ and $\mathrm{C}_{m D i V A}$ ):

$$
C_{m C T T}=\frac{2 C_{m D i V A}}{1-C_{m D i V A}} \quad \text { and } \quad C_{m D i V A}=\frac{C_{m C T T}}{2+C_{m C T T}}
$$

It is common in the literature to see a value of 0.02 for the CTT contrast threshold. The corresponding contrast threshold for DiVA would therefore be equal to.02/(2+.02) $\approx .01$. These different threshold values are important for a consistent comparison between DiVA and CTT predictions.

Comparison of CTT and DiVA maximum horizontal visibility equations: The maximum horizontal visibility, $R_{v i s}^{\max }$, given by DiVA (Maffione 2002) is:

$$
R_{v i s}^{\max }=\frac{\ln \left[\frac{K_{L}\left(C_{D i V A}(0) / C_{m D i V A}-1\right)}{2 \Lambda}+1\right]}{K_{L}},
$$

where $\Lambda$ is the "lighting factor" of DiVA. The numerator in this equation can be shown to be identical to the numerator in the CTT theory for horizontal visibility. Substituting the following relationship into the numerator

$$
\frac{2 \Lambda}{K_{L}}=\frac{2 L_{B O}}{L_{T O}+L_{B O}}=\left(1-C_{D i V A}(0)\right),
$$

the equation for maximum visibility becomes:

$$
R_{v i s}^{\max }=\frac{\ln \left[\frac{\left(C_{D i V A}(0) / C_{m D i V A}-1\right)}{1-C_{D i V A}(0)}+1\right]}{K_{L}}
$$

Making use of the previous relationships between DiVA and CTT contrasts, the bracketed expression becomes:

$$
\begin{aligned}
\frac{\left(C_{D i V A}(0) / C_{m D i V A}-1\right)}{1-C_{D i V A}(0)}+1 & =\frac{\frac{2 C_{C T T}(0)-2 C_{m C T T}}{\left(2+C_{C T T}(0)\right) C_{m C T T}}}{\frac{2}{2+C_{C T T}(0)}}+1 \\
& =\frac{C_{C T T}(0)}{C_{m C T T}}
\end{aligned}
$$

After substitution, the DiVA and CTT results can then be compared for horizontal visibility as:

$$
\left(R_{v i s}^{\max }\right)_{D i V A}=\frac{\ln \left[C_{C T T}(0) / C_{m C T T}\right]}{K_{L}} \quad \text { and } \quad\left(R_{v i s}^{\max }\right)_{C T T}=\frac{\ln \left[C_{C T T}(0) / C_{m C T T}\right]}{c}
$$

It can be seen that the main difference between DiVA and CTT is the assumption that the extinction coefficient, $c$, needs to be replaced by $K_{L}$, the radiance attenuation coefficient. The above is an important result because it shows that DiVA will, in general, predict greater maximum horizontal 
visibility than CTT since $K_{L}<c$. In particular, for a black target, with the target contrast equal to -1.0 and the CTT contrast threshold equal to -0.02 (i.e., a DiVA contrast threshold of $\sim-0.01$ ):

$$
\left(R_{v i s}^{\max }\right)_{D i V A}=\frac{3.92}{K_{L}} \quad \text { and } \quad\left(R_{v i s}^{\max }\right)_{C T T}=\frac{3.92}{c} .
$$

The above result shows that, by defining target contrast according to the Modulation Transfer Function (MTF) approach, DiVA complicates the algebra and results in intricate equations which can obscure the underlying similarities between both diver visibility algorithms.

Our analysis shows that the MTF-based definition of contrast doesn't add new information regarding contrast transmittance. However the DiVA has been put forward by HOBILabs as a "mesoscale" visibility algorithm. Mesoscale presumably implies low angular frequencies relative to the observer or camera. For example, the angular frequency corresponding to a 1 meter wide circular target at a distance of 3 meters would have a value of 3 cycles/radian. However, the same size target at a distance of 10 meters would correspond to an angular frequency of 10 cycles/radian. Historically the MTFbased definition of contrast has been reserved to treat and simulate the blurring of the higher spatial frequencies making up the finer details within a target (target detection versus identification). This blurring is due to the forward scattering of photons as they propagate from the target to the observer or camera. There is nothing inherently wrong with using the MTF-based definition of contrast for target discernment, although its use increases the complexity of the algebra without providing additional insight into the diver visibility problem. However, it is important for DiVA to determine how $K_{L}$ varies and its relationship to $c$ and $K_{a \bullet \bullet t a}$ since these are not equivalent.

Investigation of $K_{L}$ : The HOBILabs report states that $K_{L}$ is an attenuation coefficient that can be equated directly or indirectly to that measured by the a-Beta (i.e. the $K_{a \cdot-t a}$ ). The argument for the DiVA algorithm therefore rests on replacing $c$ by $K_{L}$ in addition to the MTF-based definition of contrast. This suggests that one critical aspect of DiVA rests in $K_{L}$ and its measurement by the a- $\beta$ eta. The uncertainty in determining $K_{L}$ due to target size, reflectance, and shape are all issues. This attenuation must include the radiance field and the target/background changes that occur. The DiVA model attempted to take this into account by the addition of the lighting factor, $\Lambda$. The utility of the lighting factor is an issue as demonstrated above.

The DiVA concept does make some important contributions. Diva does try to include the influence of the radiance distribution on visibility. DiVA defines a parameter $\mathrm{Q}_{T}$ as $L_{B O} / E_{T}$ as a function of $b / c$, sun angle, and look angle; where $E_{T}$ is the irradiance incident on a target. Physically, $E_{T} / \pi$ represents the radiance emanating from a white Lambertian target. This radiance contribution influence is not evident in other treatments since assumptions of asymptotic radiance distributions are made. Another contribution is the recognition that beam attenuation, $c$, is not necessarily the proper coefficient for visibility but rather this elusive $K_{L}$, which is a difficult parameter to measure as DiVA discovered.

One good illustration for selection of the appropriate visibility attenuation coefficient is for large targets that are close to the observer, but in turbid water. The effective angular frequency of the target with respect to the camera (distance/target diameter) is a critical parameter in determining if $c$ is appropriate. For turbid water with targets of reasonable size (i.e. low spatial frequency), beam $c$ can be shown to underestimate the visibility range for the target (see discussion below for 20" black target).

In-situ diver visibility analysis: In order to investigate the magnitude of $K_{L}$ with respect to $c$ and $K_{a-\bullet t a}$ several interactive GUIs were developed. Figure 1 is a GUI developed to analyze the digital images 
collected during the diver operations. With the GUI, pixel values of the background, black (or black and white) 20" target images are extracted, then normalized with respect to the background and plotted in the center graph. This data is used to fit $K_{L}$ to the data using the functional form [1- $\mathrm{e}^{-K_{L} \mathrm{R}}$ ] where $\mathrm{R}$ is the range. The graph on the right uses the target and background pixel values to derive the contrast using the CTT and DiVA formulation (blue and red curves respectively). The measured data is fit to a curve using a "contrast attenuation coefficient" Kc by changing "gamma, $\gamma$. " In DiVA, $K_{L}$ is equated to $a+\gamma b$, where $\gamma$ describes the scattering contribution to the path attenuation coefficient. Using measurements of absorption, attenuation, and $K_{a \cdot e t a}$ the $\gamma$ is calculated and then compared with the value required to fit that obtained using the images. Since there is no additional information in the DiVA formulation, both the CTT and DiVA respond the same but with a different magnitude for "contrast" as described above.

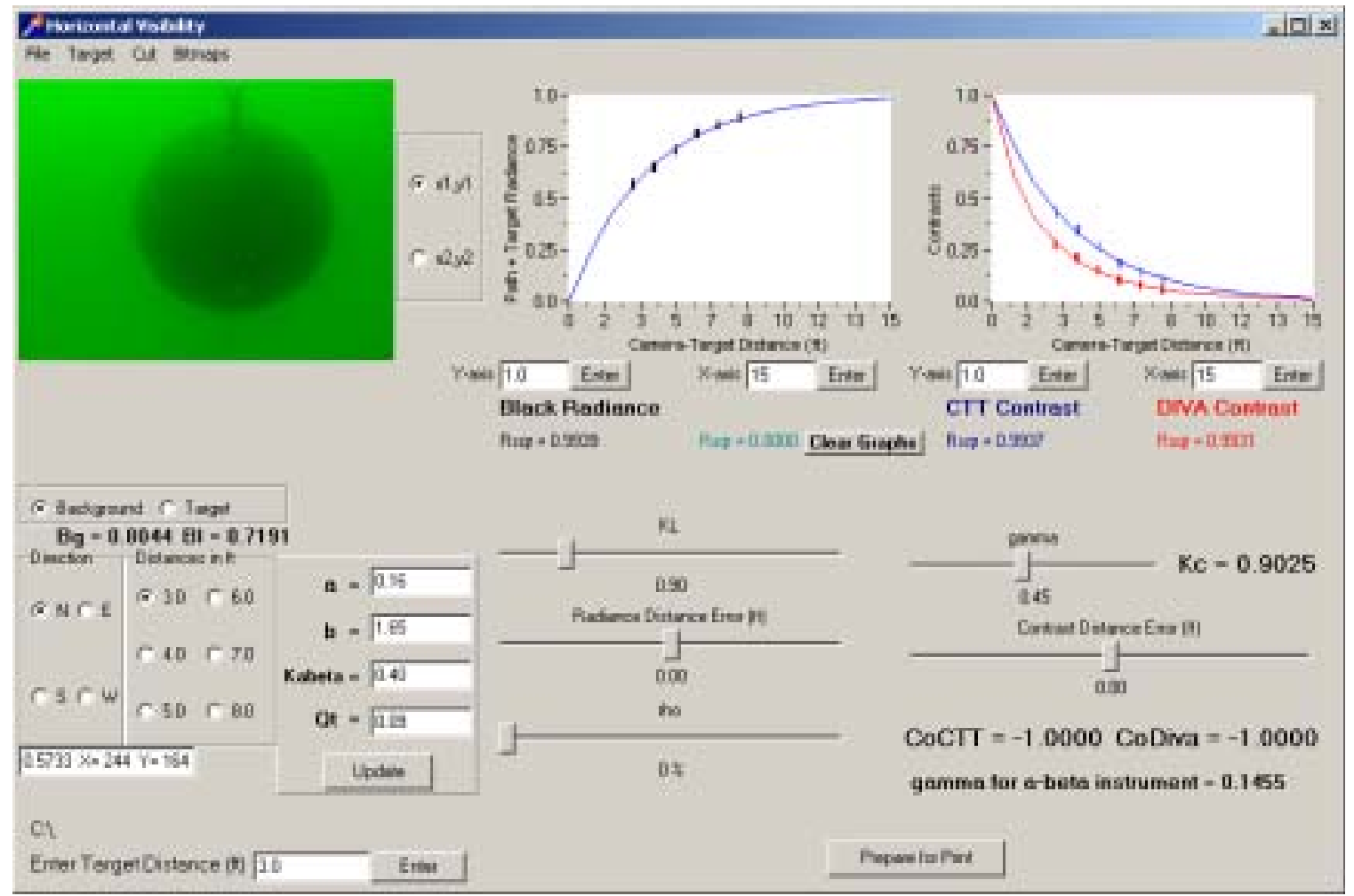

Figure 1. Graphical Interface Analysis Tool for analysis of digital imagery [Black target digital image used to determine CTT and DiVA contrasts for comparison of CTT and DiVA contrast formulations; and comparison of $K_{L}, c$, and $K_{a-e \text { etal }}$

The results using the graphical interface tool are very informative with an example from the Gulfport exercise shown above for a black target. In this example the $K_{L}$ derived from the radiances is equal to that obtained from the contrast formulations ( 0.90). It is between $a$ and $c$. Note that neither $K_{L}$ or Kc is equal to the $K_{a \cdot \bullet t a}$. Using the relationship between $K_{a \cdot \bullet t a}$ and $a$ and $b$ gives a gamma of 0.15 , whereas the gamma for the contrast is about 0.45 ; the increase is expected but the magnitude of the increase is unknown. The value of gamma and how it varies is an area of research. In other examples the Kc is very close to $c$. The importance of the variability between $K_{L}$ and $\mathrm{Kc}, c$, and $K_{a \cdot \bullet t a}$ cannot be understated. This implies that neither $c$ or $K_{a \cdot \bullet t a}$ are always the proper coefficients for large targets. Hence the variability of $K_{L}$ with respect to $c$ and $K_{a \bullet e t a}$ (and therefore gamma) is an important aspect in 
determining whether complex models for visibility add significantly to existing diver visibility algorithms. Currently, NAVOCEANO uses $4 / c$ as their standard algorithm. For black targets, VantDavies Colley found that $4.8 / \mathrm{c}$ fit their data expanding oceanic and riverine environments. For the Gulfport, MS and Pensacola, FL exercises values ranged from 3.9/c to 6.1/c (4.4 for the example above). For our test areas, the visibility could have been predicted to within about $25 \%$ using $4.8 / c$ without accounting for diver photopic response, light adaptation, radiance field, or any other of the factors affecting visibility. It has yet to be demonstrated that new models significantly improve this.

There are places in the DiVA model where some disparities arise. In running DiVA negative visibility does arise. These are probably artifacts of the numerical radiance model used and the difficulty in properly accounting for all of the interactions between the target, the observer, and the radiance field. For general Navy use such results would confuse a user about the reliability of such a model.

\section{IMPACT/APPLICATIONS}

This work will impact the selection of instrumentation and models that could potentially be incorporated into survey ships and the BattleSpace Profiler (BSP). The use of beam $c$ versus $K_{a \cdot \text { eta }}$, or a combination of these, is an important decision for the Navy. The BSP is used by Mine Warfare to provide METOC (Meteorological and Oceanographic) relevant parameters into MEDAL (Master Environmental Data Library) which is the Tactical Decision Aid used by fleet operations. The selection of a diver visibility algorithm and proper parameters is critical. If a complex model is demonstrated to work better, but with too many input parameters required, then the utility of the model is either questionable or can be used to direct other research to obtain the needed parameters.

\section{TRANSITIONS}

Presently there is no formal transition; however, the research results are being used by N85 and NAVOCEANO to determine instrumentation and models used for diver visibility.

\section{RELATED PROJECTS}

Ocean Response Coastal Analysis System. Alan Weidemann, LCDR Davis-Lunde, ONR/NOPP extension of Gauging Littoral Optics for the Warfighter and provided platform and diver support.

\section{REFERENCES}

Davies-Colley, R., 1988. "Measuring water clarity with a black disk". Limnol. Oceanogr., 33(4), pp 616-623.

Duntley, S.Q., 1963. "Light in the sea”. J. Opt. Soc. Am., vol 53, pp. 214-233.

Maffione, R., 2002. "Distance visibility algorithm”. Final Report to ONR.

\section{PUBLICATIONS}

None

\section{PATENTS}

None 